\title{
Cerebrospinal fluid antibodies to aquaporin-4 in neuromyelitis optica and related disorders: frequency, origin, and diagnostic relevance
}

Sven Jarius ${ }^{1}$, Diego Franciotta ${ }^{2}$, Friedemann Paul ${ }^{3}$, Klemens Ruprecht ${ }^{4}$, Roberto Bergamaschi ${ }^{2}$, Paulus S Rommer ${ }^{5}$, Reinhard Reuss ${ }^{6}$, Christian Probst ${ }^{7}$, Wolfgang Kristoferitsch ${ }^{8}$, Klaus Peter Wandinger ${ }^{7,9 \dagger}$, Brigitte Wildemann ${ }^{1 *+}$

\begin{abstract}
Background: In 70-80\% of cases, neuromyelitis optica (NMO) is associated with highly specific serum autoantibodies to aquaporin-4 (termed AQP4-Ab or NMO-lgG). Recent evidence strongly suggests that AQP4-Ab are directly involved in the immunopathogenesis of NMO.

Objective: To assess the frequency, syndrome specificity, diagnostic relevance, and origin of cerebrospinal fluid (CSF) AQP4-Ab in patients with NMO spectrum disorders (NMOSD).

Methods: 87 CSF samples from 37 patients with NMOSD and 42 controls with other neurological diseases were tested for AQP4-Ab in a cell based assay using recombinant human AQP4. Twenty-three paired CSF and serum samples from AQP4-Ab seropositive NMOSD patients were further analysed for intrathecal IgG synthesis to AQP4.

Results: AQP4-Ab were detectable in 68\% of CSF samples from AQP4-Ab seropositive patients with NMOSD, but in none of the CSF samples from AQP4-Ab seronegative patients with NMOSD and in none of the control samples. Acute disease relapse within 30 days prior to lumbar puncture, AQP4-Ab serum titres >1:250, and blood-CSF barrier dysfunction, but not treatment status, predicted CSF AQP4-Ab positivity. A positive AQP4-specific antibody index was present in 1/23 samples analysed.

Conclusions: AQP4-Ab are detectable in the CSF of most patients with NMOSD, mainly during relapse, and are highly specific for this condition. In the cohort analysed in this study, testing for CSF AQP4-Ab did not improve the sensitivity and specificity of the current diagnostic criteria for NMO. The substantial lack of intrathecal AQP4-Ab synthesis in patients with NMOSD may reflect the unique localisation of the target antigen at the blood brain barrier, and is important for our understanding of the immunopathogenesis of the disease.
\end{abstract}

\section{Background}

Neuromyelitis optica (NMO) is a rare inflammatory disorder of the central nervous system (CNS) that predominantly affects the optic nerves and the spinal cord [1]. Recent evidence suggests that serum antibodies to aquaporin-4 (AQP4-Ab or NMO-IgG) are directly involved in the pathogenesis of NMO [2-8]. Only very little is known to date about frequency, titres, diagnostic relevance, and pathogenic impact of AQP4- $\mathrm{Ab}$ in

\footnotetext{
* Correspondence: brigitte.wildemann@med.uni-heidelberg.de

+ Contributed equally

'Division of Molecular Neuroimmunology, Department of Neurology, University of Heidelberg, Heidelberg, Germany

Full list of author information is available at the end of the article
}

the cerebrospinal fluid (CSF) of patients with NMO and related disorders. Most previous studies investigating AQP4-Ab did not evaluate CSF, $[2,5,9-11]$ used assays with low sensitivity,[12] or did not take into account blood-CSF barrier dysfunction [13]. In particular, it is still widely unknown whether AQP4-Ab is produced intrathecally. It is also unclear whether testing of CSF for AQP4-Ab helps to diagnose NMO in patients negative for serum AQP4-Ab as suggested by a recent case report [12]. To address these issues, we tested a large series of consecutive, paired CSF and serum samples from Caucasian patients with NMO or its formes frustes, longitudinally extensive transverse myelitis (LETM) or optic neuritis (ON), as well as

\section{() Biomed Central}


from controls for AQP4-Ab using a recombinant cell based assay [14]. In contrast to a previous study,[13] we controlled for possible disturbances of the bloodCSF barrier.

\section{Patients and methods}

87 CSF samples were analysed, including 45 samples from 37 patients with NMO spectrum disorders (NMOSD) from Germany, Austria, and Italy (serum AQP4-Ab positive in 31 , negative in 14 samples) and 42 samples from control patients with multiple sclerosis (MS) or other neurological diseases (OND) (Table 1). NMOSD samples included 8 follow-up samples (AQP4-Ab seropositive in 7, seronegative in 1) from 8 different patients. 29/40 (73\%) CSF samples in the NMOSD group were obtained within 30 days from onset of the most recent clinical attack (myelitis in 17; $\mathrm{ON}$ in 8; myelitis and $\mathrm{ON}$ in 4); in 5 cases the date of relapse onset was not exactly known. Median time between onset of relapse and LP was 14 days (range, $2-640)$, and 8.5 (2-30) if only samples taken within 30 days after relapse onset were considered. $31 / 38$ samples from control patients with inflammatory disorders $(81.6 \%)$ were taken within 30 days after onset of attack; 5 were in remission at time of LP; and 2 had chronic progressive disease. Median disease duration was 19.4 months (range, 0.1-286) in the NMOSD group and 19.3 (0.1-143.5) in the MS group. Lumbar puncture was done for diagnostic purposes in all cases. Samples were stored at $-80^{\circ} \mathrm{C}$ until tested. All samples were tested in a cell based assay (CBA) as previously described [14]. This assay was previously demonstrated to have a sensitivity of $78 \%$ for NMO and a specificity of 100\% [14]. From 23 paired CSF and serum samples sufficient material was available for assessment of the $\mathrm{AQP} 4$-specific antibody index (AI), $\mathrm{AI}_{\mathrm{AQP} 4}$. Calculation of AIs allows quantification of antigen-specific intrathecal antibody synthesis $[15,16]$. Briefly, $\mathrm{AI}_{\mathrm{AQP} 4}$ values were calculated as the ratio between the CSF/ serum quotient for AQP4-IgG, $\mathrm{Q}_{\mathrm{AQP} 4}$, and the CSF/ serum quotient for total IgG, $\mathrm{Q}_{\mathrm{IgG}}$; i.e., $\mathrm{AI}_{\mathrm{AQP} 4}=$ $\mathrm{Q}_{\mathrm{AQP} 4} / \mathrm{Q}_{\mathrm{IgG}}$. If $\mathrm{AQP} 4-\mathrm{IgG}$ are produced intrathecally, $\mathrm{Q}_{\mathrm{AQP} 4}$ would exceed $\mathrm{Q}_{\mathrm{IgG}}$, resulting in AI values $>1$. Usually, values $>1.5$ are considered as evidence of intrathecal specific antibody synthesis $[15,16]$. However, if titres instead of concentrations are used to calculate the AI, a cut-off of 4 has been recommended [17]. Reiber's empiric hyperbolic function $\mathrm{Q}_{\text {lim }}$ was applied to control for possible underestimation of intrathecal specific synthesis due to disturbances of the blood-CSF barrier function:[18]

$$
\mathrm{Q}_{\lim (\operatorname{IgG})}=0.93 \sqrt{\left(\mathrm{Q}_{\mathrm{Alb}}\right)^{2}+6 \times 10^{-6}}-1.7 \times 10^{-3}
$$

In case of $\mathrm{Q}_{\mathrm{IgG}}>\mathrm{Q}_{\lim (\mathrm{IgG})}, \mathrm{AI}_{\mathrm{AQP} 4}$ was calculated as the ratio between $\mathrm{Q}_{\mathrm{AQP} 4}$ and $\mathrm{Q}_{\lim (\mathrm{IgG})}$, i.e., $\mathrm{AI}_{\mathrm{AQP} 4}=$ $\mathrm{Q}_{\mathrm{AQP4}} / \mathrm{Q}_{\lim (\mathrm{IgG})}$. For assessment of the AI, serum samples were tested at 1:10, 1:100, 1:500, 1:1000, and 1:5000 dilutions, and at 1:25, 1:50, 1:62.5, 1:75, 1:125, 1:250, $1: 750,1: 1250,1: 1750,1: 2000,1: 2500,1: 3000,1: 4000$, $1: 6000,1: 7000,1: 8000,1: 9000$, and 1:10000 dilutions, where applicable. CSF samples were tested undiluted, at 1:10 dilution, and, in addition, at dilutions that would indicate intrathecal production as defined by an elevated AQP4-AI of $>4$.

Values for $\mathrm{Q}_{\mathrm{IgG}}$ exceeding the hyperbolic discrimination line, $\mathrm{Q}_{\text {lim }}$, or detection of CSF-restricted oligoclonal bands (OCB) (data taken from the patient records), were considered as indicative of intrathecal synthesis of total IgG (as opposed to AQP4-specific IgG) [16]. The $\mathrm{CSF} /$ serum albumin ratio, $\mathrm{Q}_{\mathrm{Alb}}=\mathrm{Alb}_{\mathrm{CSF}}[\mathrm{mg} / \mathrm{l}] / \mathrm{Alb}_{\text {serum }}$ $[\mathrm{g} / \mathrm{l}]$, was used to assess the blood-CSF barrier function. The upper reference limit of $\mathrm{Q}_{\mathrm{Alb}}$ was calculated as 4 $+(a / 15)$ with $a$ representing the patient's age [19].

Table 1 Epidemiological data and sample numbers

\begin{tabular}{|c|c|c|c|c|c|c|c|}
\hline & $\begin{array}{l}\text { Number of } \\
\text { patients }\end{array}$ & Caucasian & $\begin{array}{l}\text { Sex ratio, male: } \\
\text { female }\end{array}$ & $\begin{array}{l}\text { Relapsing } \\
\text { course }\end{array}$ & $\begin{array}{l}\text { Paired CSF/serum } \\
\text { samples }\end{array}$ & $\begin{array}{l}\text { Median age at LP } \\
\text { (range) }\end{array}$ & $\begin{array}{l}\text { Untreated at time of } \\
\text { LP (\%) }\end{array}$ \\
\hline Total & 79 & $72 / 79(91)$ & $1: 4.6$ & $59 / 79(75)$ & 87 & $40(15-72)$ & $56 / 80(70)$ \\
\hline NMOSD & 37 & $32 / 37$ (87) & $1: 17.5$ & 33/37 (89) & 45 & $41(17-72)$ & 19/40 (48)† \\
\hline Controls & 42 & $40 / 42(95)$ & $1: 2.8$ & $26 / 42(62)$ & 42 & $39(15-70)$ & $37 / 40$ (93)‡ \\
\hline MS & 28 & $26 / 28(92)$ & $1: 2.1$ & 26/28 (93) & 28 & $38(15-69)$ & $2 / 27$ \\
\hline OND & 14 & $\begin{array}{l}14 / 14 \\
(100)\end{array}$ & $1: 3.7$ & $0 / 14(0)$ & 14 & $45(20-70)$ & $1 / 13$ \\
\hline
\end{tabular}

Diagnoses in the MS group included relapsing-remitting MS in 26; secondary progressive MS in 1; and primary progressive MS in 1. Diagnoses in the OND group included acute demyelinating encephalomyelitis in 4; non-longitudinally extensive transverse myelitis in 1; brain stem encephalitis of unknown aetiology in 1; autoimmune cerebellitis in 1; Herpes simplex virus encephalitis in 1; CNS lymphoma in 1; primary angiitis of the CNS in 1; Behçet's disease in 1; benign paroxysmal positional vertigo in 1; hydrocephalus aresorptivus in 1; and spinal disc prolaps in 1. NMO was diagnosed according to reference [39]. LETM was defined as myelitis extending over three or more segments as demonstrated by magnetic resonance imaging. MS was diagnosed according to reference [40]. $\dagger$ Treatments in the remaining cases included oral steroids, intravenous methylprednisolone, azathioprine, methotrexate, and cyclo-phosphamide; in 5 cases no exact data on the treatment status at time of LP were available. $\neq$ In 2 cases, no exact data on the treatment status was available. LETM = longitudinally extensive transverse myelitis; MS = multiple sclerosis; $\mathrm{NMO}=$ neuromyelitis optica; $\mathrm{n} . \mathrm{d}$. = not determined; NMOSD = neuromyelitis spectrum disorders; ON = optic neuritis; OND = other neurological diseases 


\section{Results}

\section{AQP4-Ab CSF status}

AQP4-IgG was detected in 21/31 (68\%) CSF samples from AQP4-Ab seropositive NMOSD patients. 0/14 CSF samples from AQP4-IgG seronegative NMOSD patients and 0/42 control samples were positive for AQP4-IgG. No significant difference regarding CSF AQP4-Ab frequency was found between acute relapses of ON (75\%) and myelitis (59\%). Detailed results are given in Table 2.

\section{AQP4-Ab serum titres}

Serum AQP4-IgG titres were determined in 26/31 NMOSD samples and were higher (median 1:1000; range, 1:250-1:12.500) in CSF AQP4-IgG-positive patients $(\mathrm{n}=18)$ than in CSF AQP4-IgG-negative patients $(1: 250 ; 1: 10-1: 1000 ; \mathrm{n}=8)$ ( $\mathrm{p}<0.002$; MannWhitney test). See Figure 1 and Table 2 for details.

\section{Blood-CSF barrier function}

Disturbances of the blood-CSF barrier as indicated by elevated QAlb were present in 11/18 (61\%) AQP4-Ab serum and CSF positive NMOSD samples but only in 1/ 8 (13\%) of the AQP4-Ab seropositive but CSF-negative NMOSD samples analysed ( $\mathrm{p}=0.036$; Fisher exact test). In the AQP4-Ab seronegative group, blood-CSF barrier dysfunction was present in $6 / 9(67 \%)$ cases. In the remaining cases not enough CSF or serum was available for $\mathrm{Q}_{\mathrm{Alb}}$ determination. No significant correlation between AQP4-Ab titres and $Q_{\text {Alb }}$ was found.

Total IgG in the CSF and serum

Evidence for intrathecal synthesis of total IgG as indicated by either CSF-restricted OCBs or elevated $\mathrm{Q}_{\mathrm{IgG}}$ was present in $32.5 \%(13 / 40)$ NMOSD, and was slightly

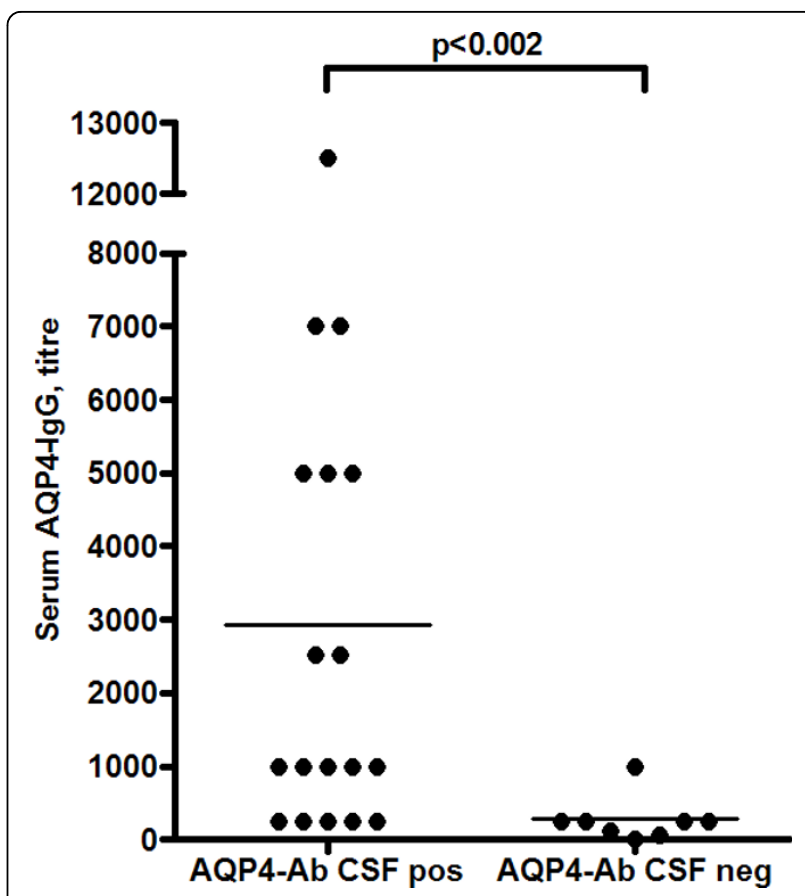

Figure 1 Distribution of AQP4-IgG serum titres according to AQP4-Ab CSF status. AQP4-Ab IgG serum titres were significantly higher in AQP4-Ab CSF-positive patients.

more frequent in AQP4-Ab seronegative NMOSD samples $(50 \%$, or $6 / 12)$ compared to seropositive samples (25\%, or $7 / 28)$. No significant correlation with the AQP4-Ab CSF status was found; while 100\% (7/7) of the samples with intrathecally produced total IgG that were obtained from AQP4-Ab seropositive patients were positive for CSF AQP4-Ab, also 57.1\% (12/21) of the CSF samples from AQP4-Ab seropositive patients with

Table 2 Clinical findings, AQP4-Ab status in serum and CSF, and median AQP4-Ab serum titres in the various disease groups

\begin{tabular}{llllll}
\hline Diagnosis & $\begin{array}{l}\text { No of CSF } \\
\text { samples }\end{array}$ & $\begin{array}{l}\text { Acute attack at time of LP } \\
(\%)\end{array}$ & $\begin{array}{l}\text { AQP4-Ab, serum } \\
(\%)\end{array}$ & $\begin{array}{l}\text { AQP4-Ab, CSF } \\
(\%)\end{array}$ & $\begin{array}{l}\text { Median serum titre (range; } \\
\text { N) }\end{array}$ \\
\hline NMOSD & 45 & $29 / 40(73)$ & $31 / 45(69)$ & $21 / 45(47)$ & $1000(10-12500 ; 26)$ \\
NMO & 26 & $16 / 22(73)$ & $16 / 26(62)$ & $11 / 26(42)$ & $1000(125-7000 ; 12)$ \\
LETM & 8 & $6 / 8(75)$ & $7 / 8(88)$ & $5 / 8(63)$ & $250(62.5-12500 ; 7)$ \\
ON & 11 & $7 / 10(70)$ & $8 / 11(73)$ & $5 / 11(45)$ & $250(10-7000 ; 7)$ \\
Relapse & 29 & $29 / 29(100)$ & $20 / 29(69)$ & $17 / 29(59)$ & $1000(250-12500 ; 20)$ \\
$\quad \begin{array}{l}\text { Remission } \\
\text { AQP4-Ab }\end{array}$ & 11 & $0 / 11(0)$ & $7 / 11(64)$ & $1 / 11(9)$ & $187.5(10-250 ; 6)$ \\
seropositive & 31 & $20 / 31(65)$ & $31 / 31(100)$ & $21 / 31(68)$ & $1000(10-12500 ; 26)$ \\
$\quad \begin{array}{l}\text { AQP4-Ab } \\
\text { seronegative }\end{array}$ & 14 & $9 / 14(64)$ & $0 / 14(0)$ & $0 / 14(0)$ & Negative \\
Controls & 42 & $31 / 38(82)$ & $0 / 42(0)$ & $0 / 42(0)$ & Negative \\
$\quad$ MS & 28 & $22 / 28(79)$ & $0 / 28(0)$ & $0 / 28(0)$ & Negative \\
OND & 14 & $9 / 10(90)^{5}$ & $0 / 14(0)$ & $0 / 14(0)$ & Negative \\
\hline
\end{tabular}

AQP4-Ab = aquaporin-4 antibody; CSF = cerebrospinal fluid; LETM = longitudinally extensive transverse myelitis; MS = multiple sclerosis; NMOSD = NMO spectrum disorders; NMO = neuromyelitis optica; ON = optic neuritis; OND = other neurological diseases. ${ }^{\S}$ Not applicable in four patients with non-inflammatory neurological diseases. 
Table 3 Clinical and laboratory features according to AQP4-Al status

\begin{tabular}{lll}
\hline & AQP4-Ab Al pos & AQP4-Ab Al neg \\
\hline No of CSF samples & 1 & 22 \\
Sex & Female & All female \\
Age & 22 & $40(14-72)$ \\
Diagnosis at time of LP & rON* & NMO in 11; LETM in 6; ON in 5 \\
IS/IM treatment at time of LP & Steroids until 5 d before LP & $11 / 22$ \\
Disease duration in months, median & 19.4 & $20.5(0.1-120)$ \\
Acute attack at time of LP & Yes & $19 / 22$ \\
AQP4-Ab serum titre, median (range) & $1: 1000$ & $1000(250-12500)$ \\
QlgG, median (range) & 5.85 & $4.8(1.5-16.1)$ \\
Intrathecal synthesis of total lgG & Yes & $6 / 22$ \\
QAlb, median (range) & 7.17 & $6.7(3.2-31.1)$ \\
Blood-CSF barrier dysfunction & Yes & $11 / 22$ \\
\hline
\end{tabular}

* Developed NMO 10 months later. AI = antibody index; IS/IM = immunosuppressive/immunomodulatory; NMO = neuromyelitis optica; LETM = longitudinally extensive transverse myelitis; LP = lumbar puncture; QAlb = albumin CSF/serum ratio; QlgG = lgG CSF/serum ratio; rON = recurrent optic neuritis.

neither OCBs nor $\mathrm{Q}_{\mathrm{IgG}}$ elevation harboured AQP4-Ab $\left(\mathrm{p}=\right.$ n.s.; Fisher exact test). Also, $\mathrm{Q}_{\mathrm{IgG}}$ and AQP4-Ab titres showed no significant correlation.

\section{AQP4-Ab AI}

Intrathecal production (IP) of AQP4-IgG as defined by an AI $>4$ was found in only $1 / 23$ NMOSD samples analysed $(4.3 \%)\left(\mathrm{AI}_{\mathrm{AQP} 4}=7\right)$. This patient had experienced a third attack of ON 12 days prior to LP (first LETM 10 months later) and had received high-dose methylprednisolone until nine days and low-dose steroids until five days prior to LP. The patient had co-existing acetylcholine receptor-antibody positive myasthenia gravis (treated with pyridostigmine) and thyroglobulin and thyroid peroxidase antibody positive thyroiditis. Clinical and laboratory findings at time of LP were otherwise unremarkable when compared to the remaining patients (Table 3). The sample was positive for OCBs, and $\mathrm{Q}_{\mathrm{IgG}}\left(5.9 ; \mathrm{Q}_{\lim (\mathrm{IgG})}=5.3\right.$; intrathecal IgG fraction, 8.7\%) and $\mathrm{Q}_{\mathrm{Alb}}$ (7.2; age-adjusted upper reference limit $=5.5$ ) were both elevated. A followup sample taken from the same patient one year later during another relapse of $\mathrm{ON}$ and still prior to initiation of long-term immunosuppression did not show evidence of AQP4-Ab IP anymore, though the antibody was still detectable in the CSF. In the remaining cases, not enough CSF and serum for $\mathrm{Q}_{\mathrm{IgG}}$ and $\mathrm{Q}_{\mathrm{Alb}}$ determination was available, so that AQP4-AI could not be assessed $(n=5)$, or CSF titres that would indicate a positive AQP4-AI were below $1: 1(n=3)$, so that testing was not possible (all of the latter samples were negative at 1:1 dilution).

\section{Impact of disease activity}

17/20 (85\%) CSF samples from AQP4-Ab seropositive NMOSD patients obtained within 30 days after onset of relapse were AQP4-Ab positive but only $1 / 7$ (14.3\%) taken during remission $(\mathrm{p}=0.0017$; Fisher exact test, 2 tailed). Similarly, 17/18 (94.4\%) samples positive for CSF and serum AQP4-Ab were taken during acute relapse; by contrast, only 3/9 (33.3\%) AQP4-Ab CSF-negative but AQP4-Ab seropositive samples were obtained during relapse $(\mathrm{p}=0.002$; Fisher exact test). The median serum AQP4-Ab titre of those NMSOD samples taken during relapse (1:1000; range, 1:250-1:12500; $\mathrm{n}=20$ ) was higher than in NMOSD samples obtained during remission $(1: 187.5 ; 1: 10-1: 250 ; \mathrm{n}=6)(\mathrm{p}=0.0008$; Mann-Whitney test). AQP4-Ab serum titres did not differ markedly between acute relapses of myelitis (median, $1: 1000 ; \mathrm{n}=13$ ) and $\mathrm{ON}$ (median, 1:2500; $\mathrm{n}=6$ ), and were relatively high in the only patient with acute relapse of simultaneous optic neuritis and myelitis (1:5000). Serum AQP4-Ab titre in the single patient with evidence for AQP4-Ab IP was 1:1000. IP of total IgG was found in 7/20 (35\%) AQP4-Ab seropositive samples obtained during relapse but in $0 / 7$ taken during remission. Disruption of the blood-CSF barrier was found both with AQP4-IgG seropositive samples taken during relapse $(10 / 20 ; 50 \%)$ and with some of those obtained during remission $(2 / 6 ; 33 \%)$. No significant differences between acute relapses of myelitis and acute relapses of $\mathrm{ON}$ were found regarding the frequency of total IgG IP or of blood-CSF barrier disruption.

\section{Impact of disease duration}

Median disease duration at time of LP was shorter (24.5 $\mathrm{v} 4.1$ months) in the AQP4-Ab seronegative group ( $\mathrm{p}=$ 0.056; Mann-Whitney test). Among seropositive patients, those positive for CSF AQP4-Ab had a longer disease duration (52.8 vs 19.45 months; $\mathrm{p}=0.041$ ). No correlation between serum titres and disease duration or time since relapse onset was found.

\section{Impact of treatment status}

Median serum AQP4-Ab titres did not differ significantly between untreated $(n=12 ; 1: 625$; range, 1:62.5- 
$1: 12500)$ and treated $(\mathrm{n}=14 ; 1: 1000 ; 1: 10-1: 7000)$ NMOSD patients nor did the AQP4-IgG CSF positivity rate $(64.3 \%$ and $75 \%$, respectively). The only patient with positive $\mathrm{AI}_{\mathrm{AQP}} 4$ had received steroids until five days before LP.

\section{Longitudinal analysis}

In total, 8 follow-up samples from 8 patients with NMOSD were examined for AQP4-Ab (median latency, 381 days). No patient who was initially positive for serum AQP4-Ab was negative at follow up; the only patient negative at first testing was also negative at follow-up. However, the disease status (relapse or remission) at first and second LP was identical in all cases. Also, in all cases, CSF was positive for AQP4-Ab in both samples, if samples were taken during relapse, or negative, if samples were taken during remission; the only exception was one AQP4-Ab seropositive sample that was negative for CSF AQP4-Ab during a relapse of $\mathrm{ON}$ but positive during a relapse of myelitis.

\section{Discussion}

In this study we systematically evaluated the frequency of AQP4-IgG in the CSF and serum of Caucasian patients with NMOSD. Using a CBA employing recombinant human AQP4, we found CSF AQP4-IgG in 70\% of AQP4-IgG seropositive NMOSD samples, but in none of the MS or OND controls.

AQP4-IgG CSF positivity was associated with higher AQP4-IgG serum titres and with dysfunction of the blood-CSF barrier. Moreover, AQP4-IgG was more frequently detectable during relapse $(\mathrm{p}=0.0017)$. The latter finding most likely reflects an increase in serum AQP4-IgG titres during relapse, since no evidence of intrathecally produced AQP4-IgG was found in almost all cases analysed. Serum titres were indeed significantly higher during relapse than in remission in our patients (median, $1: 1000$ vs $1: 187.5)(\mathrm{p}=0.001)$, which is in line with previous studies [13,20]. Interestingly, the cut-off serum AQP4-IgG titre that predicted CSF AQP4-IgG positivity (1:250) was similar to that found in Japanese patients with opticospinal MS [13].

Serum titres $>1: 250$ were associated with acute disease in all cases, and titres $<1: 250$ with remission. In contrast, no clear correlation was found in case the serum titre was 1:250. It should be mentioned as a caveat, however, that the number of samples obtained during remission was relatively low in this study (as LP is mainly done for acute disease). In a previous study that included samples obtained over a period of up to five years, we could demonstrate marked variations over time regarding AQP4-Ab concentrations during remission with no general cut-off for relapse induction (though relapses were always preceded by an relative increase in AQP4-Ab levels). The latter finding might indicate differences in AQP4-Ab affinity and specificity between patients and over time, but inter- and intraindividual variations regarding $\mathrm{T}$-cell activation, cytokine levels, or BBB function may also play a role.

Serum AQP4-Ab titres did not differ markedly between untreated and treated NMOSD patients nor did the AQP4-IgG CSF-positivity rate. This could be due to the fact that $11 / 14$ samples from treated patients were taken during relapse, which was more commonly associated with high serum AQP4-Ab titres as well as with CSF AQP4-Ab.

Intrathecal AQP4-Ab production was present in only 1 out of 23 samples studied (4.3\%). This sample was obtained during an acute relapse of ON. However, 20/ 23 AQP4-IgG CSF-positive samples with normal $\mathrm{AI}_{\mathrm{AQP} 4}$ values were also taken during relapse. $\mathrm{AI}_{\mathrm{AQP} 4}$ elevation seems thus not a suitable disease activity marker. The infrequency of AQP4-IgG IP suggests that in patients with NMOSD AQP4-Ab producing B cell clones usually reside in the systemic compartment. CSF AQP4-Ab might thus reflect passive diffusion of serum AQP4-Ab into the CSF. Accordingly, Takahashi et al. (2007), in a study on 12 Japanese patients, found that titres of CSF AQP4-IgG were almost proportional to serum AQP4IgG in NMO, though, as a limitation, that study had not taken into account possible blood-CSF barrier disruption [13].

Although intrathecal AQP4-Ab production would then not be a prerequisite for inflammation in NMO, the single patient with a positive AI in our series (as well as a second recently published case with a slightly elevated $\mathrm{AI}[8]$ ) indicates that intrathecal AQP4-Ab production can occur in NMOSD. However, the rarity with which it was detected argues against a major pathogenic function.

Unlike in MS, intrathecal total IgG synthesis in NMO does not persist over time [21-24]. It is of note that evidence for total IgG IP was present in $35 \%$ of seropositive samples obtained during relapse but in none of the seropositive samples obtained during remission. In line with this finding, Melamud et al., in a study so far only published as abstract, reported intrathecal total IgG synthesis in $45 \%$ of samples obtained in bout versus $0 \%$ in remission [24]. This might explain the low frequency of intrathecal total IgG production (17-33\%) reported in the literature [1,21-23,25] compared to MS (95-100\%). Similarly, AQP4-Ab might be present in the CSF only transiently as indicated by our finding of a higher AQP4-Ab CSF positivity rate during relapse. It is of note, however, that all eleven samples obtained at approximately $(+/-8$ days $)$ the same time from onset of relapse than the only AI positive sample (which was taken at day 12 after relapse onset), showed a negative 
$\mathrm{AI}$, rendering it unlikely that timing issues are of high importance.

In our cohort, all CSF AQP4-IgG positive samples were also positive for serum AQP4-IgG, and AQP4-Ab serum titres were higher than AQP4-Ab CSF titres in all cases analysed. This would indicate that testing of CSF for AQP4-Ab in addition to serum AQP4-IgG testing is of limited benefit. In contrast, Klawiter et al. recently described three patients positive for AQP4-Ab in the CSF but not in the serum [12]. However, some constraints apply. First, a 1:128 serum dilution was used to test for AQP4-IgG instead of the standard 1:60 starting dilution $[2,4,26,27]$. To our experience from longitudinal studies in AQP4-IgG positive patients[20], serum samples may be positive even only at 1:10 dilution, depending on disease activity status or treatment. Secondly, the method used to test for AQP4-IgG, immunohistochemistry (IHC), has been demonstrated to be less sensitive compared to recombinant assays $[9,13,14]$. Thirdly, the authors discuss that interfering auto-antibodies could have led to false-negative results, a problem inherent to the IHC assay but not to the CBA. Therefore, we speculate that those samples might have been positive if a more sensitive and specific assay as well as lower serum dilutions had been applied.

The concept that pathogenic effects in NMOSD are predominantly brought about by blood derived AQP4$\mathrm{Ab}$ is compatible with the unique localisation of the $\mathrm{AQP} 4$ antigen. AQP4 is mainly expressed in the astrocytic endfeet at the glial-endothelial interface [3]. Local BBB disturbances would thus be sufficient to render the antigen directly accessible to serum antibodies. In a recent animal study, induction of BBB damage by pretreatment with complete Freund's adjuvans was in fact sufficient to cause NMO-like lesions following passive transfer of AQP4-Ab positive sera [28].

However, severe BBB damage might not even be a prerequisite for lesion formation. The pial cell layer that separates the perivascular Virchow-Robin space of larger vessels from the glia limitans is fenestrated at the level of the arterioles and is widely missing at the level of capillaries, venules, and veins. In these areas, the AQP4expressing astrocytic endfeet are directly exposed to antibodies leaving the microvasculature [29]. Importantly, the perivascular and subpial spaces harbour all cellular components required to mount local immune responses [30,31]. Moreover, serum AQP4-IgG could gain access to the CSF in the absence of $\mathrm{BBB}$ damage via regions of physiologically increased $\mathrm{BBB}$ permeability such as the circumventricular organs or by extracellular pathways, which provide a quasi-equilibrium between plasma and the fluids of the CNS $[30,32,33]$. In an animal model of NMO, injection of AQP4-Ab positive sera and human complement into the CSF indeed resulted in generation of NMO-like lesions in the absence of $\mathrm{BBB}$ disruption or pre-existing CNS inflammation [34]. In our study, seven patients had in fact normal $Q_{\text {Alb }}$ values but suffered from an acute attack, had high serum AQP4-Ab titres $>=1: 250$ (median, 1:1000), and were positive for CSF AQP4-Ab at time of LP. However, as a limitation, three of those patients were treated at time of LP, and in three the attack had started already 23, 28 and 30 days before LP. Moreover, $\mathrm{Q}_{\mathrm{Alb}}$ might not be sufficiently sensitive to reflect locally restricted BBB disruption, in particular in $\mathrm{ON}$, which was present in 4 of the 7 patients at time of LP.

Finally, AQP4-Ab itself could induce BBB damage. Prolonged exposure to AQP4-IgG in the fenestrated perivascular and subpial spaces could lead to BBB disruption by gradual local inflammation or AQP4 internalisation, followed by exacerbation of the autoimmune response [30]. A number of recent in vitro and in vivo studies revealed that IgG from patients with NMO initiates endocytosis of AQP4, a process which alters the polarized expression pattern of AQP4 on the plasma membrane and, as a functional consequence, increases BBB permeability in vitro [35-37]. In AQP4 knock-out mice, the lack of AQP4 is mirrored by tight junction opening in brain microvessels, swelling of perivascular astrocytic processes, and BBB hyperpermeability [38]. Interestingly, disease activity was linked to higher CSF $\mathrm{AQP} 4-\mathrm{Ab}$ and to higher serum AQP4-Ab levels in this study and another,[13] indicating that CSF and/or serum AQP4-IgG exceeding a threshold value might be required to induce clinically relevant inflammation.

\section{Conclusion}

In summary, our study demonstrates that (1) AQP4-IgG is detectable in the CSF in most AQP4-IgG seropositive NMOSD patients but not in that of patients with MS or OND; (2) that the presence of CSF AQP4-IgG in patients with NMOSD is positively associated with acute disease relapse within 30 days prior of LP; AQP4-IgG serum titres >1:250; and with blood-CSF barrier disruption; but not with treatment status or the type of acute clinical disease (myelitis or ON) at time of LP; (3) a lack of quantitative evidence for intrathecal synthesis of AQP4-IgG in most NMOSD patients. Our findings argue against the need to test CSF for AQP4-Ab if the corresponding serum is negative for the antibody or AQP4-Ab serum titres are below 1:250. Moreover, our results suggest that intrathecal production of $\mathrm{AQP} 4-\mathrm{Ab}$ may not be a prerequisite of disease activity in NMO.

\section{Acknowledgements}

This work was supported by a Research Fellowship from the European Committee for Treatment and Research in Multiple Sclerosis (ECTRIMS) to SJ, by grants from Bayer Schering Pharma and from Merck Serono to SJ and 
BW, and by a grant from the German Research Foundation (DFG, Cluster of Excellence 257) to FP. We are grateful to Mrs Anna Eschlbeck, Mrs Brigitte Fritz, and the Nikon Imaging Center, University of Heidelberg, for excellent technical assistance.

\section{Author details}

'Division of Molecular Neuroimmunology, Department of Neurology, University of Heidelberg, Heidelberg, Germany. ${ }^{2}$ RCCS, Foundation "Neurological Institute C. Mondino", University of Pavia, Pavia, Italy. ${ }^{3}$ NeuroCure Clinical Research Center, Charité - University Medicine Berlin, Berlin, Germany. ${ }^{4}$ Department of Neurology, Charité - University Medicine Berlin, Berlin, Germany. ${ }^{5}$ Department of Neurology, Medical University of Vienna, Vienna, Austria. ${ }^{6}$ Department of Neurology, Hospital Hohe Warte, Bayreuth, Germany. Institute for Experimental Immunology, affiliated to Euroimmun, Luebeck, Germany. ${ }^{8}$ Department of Neurology,

Sozialmedizinisches Zentrum Ost - Donauspital, Vienna, Austria. ${ }^{9}$ Institute for Neuroimmunology and Clinical MS Research, University Medical Center Eppendorf, Hamburg, Germany.

\section{Authors' contributions}

SJ conceived and designed the study. SJ, CP, and KPW were involved in carrying out the immunoassays. SJ, DF, FP, KR, RB, PR, RR, WK, KPW, and BW participated in CSF and data collection. SJ performed the statistical analysis and wrote the initial draft. SJ, DF, FP, KR, RB, PR, RR, WK, KPW, and BW participated in the preparation of the manuscript. All authors read and approved the final version of the manuscript.

\section{Competing interests}

$\mathrm{SJ}, \mathrm{DF}, \mathrm{FP}, \mathrm{KR}, \mathrm{RB}, \mathrm{PR}, \mathrm{RR}, \mathrm{WK}$, and $\mathrm{BW}$ declare no competing interests. The cells used in this study were kindly provided by Euroimmun, Luebeck, Germany. KPW and CP are employees of Euroimmun. Euroimmun had no role in study design, data collection or analysis, preparation of the manuscript, or decision to publish.

Received: 2 September 2010 Accepted: 8 September 2010 Published: 8 September 2010

\section{References}

1. Wingerchuk DM, Hogancamp WF, O'Brien PC, Weinshenker BG: The clinical course of neuromyelitis optica (Devic's syndrome). Neurology 1999, 53:1107-1114.

2. Lennon VA, Wingerchuk DM, Kryzer TJ, Pittock SJ, Lucchinetti CF, Fujihara K, Nakashima I, Weinshenker BG: A serum autoantibody marker of neuromyelitis optica: distinction from multiple sclerosis. Lancet 2004, 364:2106-2112.

3. Jarius S, Paul F, Franciotta D, Waters P, Zipp F, Hohlfeld R, Vincent A, Wildemann B: Mechanisms of Disease: aquaporin-4 antibodies in neuromyelitis optica. Nat Clin Pract Neurol 2008, 4:202-214.

4. Lennon VA, Kryzer TJ, Pittock SJ, Verkman AS, Hinson SR: IgG marker of optic-spinal multiple sclerosis binds to the aquaporin-4 water channel. J Exp Med 2005, 202:473-477.

5. Jarius $S$, Franciotta $D$, Bergamaschi $R$, Wright $H$, Littleton E, Palace J, Hohlfeld R, Vincent A: NMO-IgG in the diagnosis of neuromyelitis optica. Neurology 2007, 68:1076-1077.

6. Bradl M, Misu T, Takahashi T, Watanabe M, Mader S, Reindl M, Adzemovic M, Bauer J, Berger T, Fujihara K, et al: Neuromyelitis optica: Pathogenicity of patient immunoglobulin in vivo. Ann Neurol 2009, 66:630-643

7. Jarius S, Wildemann B: AQP4 antibodies in neuromyelitis optica: pathogenic and diagnostic relevance. Nature Reviews Neurology 2010, 6:383-392.

8. Bennett JL, Lam C, Kalluri SR, Saikali P, Bautista K, Dupree C, Glogowska M, Case D, Antel JP, Owens GP, et al: Intrathecal pathogenic anti-aquaporin-4 antibodies in early neuromyelitis optica. Ann Neurol 2009, 66:617-629.

9. Waters P, Jarius $S$, Littleton E, Leite MI, Jacob S, Gray B, Geraldes R, Vale T, Jacob A, Palace J, et al: Aquaporin-4 antibodies in neuromyelitis optica and longitudinally extensive transverse myelitis. Arch Neurol 2008, 65:913-919.

10. Paul F, Jarius S, Aktas $\mathrm{O}$, Bluthner M, Bauer $\mathrm{O}$, Appelhans $\mathrm{H}$, Franciotta D, Bergamaschi R, Littleton E, Palace J, et al: Antibody to aquaporin 4 in the diagnosis of neuromyelitis optica. PLoS Med 2007, 4:e133.
11. Hayakawa S, Mori M, Okuta A, Kamegawa A, Fujiyoshi Y, Yoshiyama Y, Mitsuoka K, Ishibashi K, Sasaki S, Hattori T, Kuwabara S: Neuromyelitis optica and anti-aquaporin-4 antibodies measured by an enzyme-linked immunosorbent assay. J Neuroimmunol 2008, 196:181-187.

12. Klawiter EC, Alvarez E, Xu J, Paciorkowski AR, Zhu L, Parks BJ, Cross AH, Naismith RT: NMO-IgG detected in CSF in seronegative neuromyelitis optica. Neurology 2009, 72:1101-1103.

13. Takahashi T, Fujihara K, Nakashima I, Misu T, Miyazawa I, Nakamura M, Watanabe S, Shiga Y, Kanaoka C, Fujimori J, et al: Anti-aquaporin-4 antibody is involved in the pathogenesis of NMO: a study on antibody titre. Brain 2007, 130:1235-1243.

14. Jarius S, Probst C, Borowski K, Franciotta D, Wildemann B, Stoecker W, Wandinger KP: Standardized method for the detection of antibodies to aquaporin-4 based on a highly sensitive immunofluorescence assay employing recombinant target antigen. J Neurol Sci 2010, 291:52-56.

15. Reiber $H$, Ungefehr $\mathrm{S}$, Jacobi $\mathrm{C}$ : The intrathecal, polyspecific and oligoclonal immune response in multiple sclerosis. Mult Scler 1998, 4:111-117.

16. Reiber $\mathrm{H}$ : Cerebrospinal fluid-physiology, analysis and interpretation of protein patterns for diagnosis of neurological diseases. Mult Scler 1998, 4:99-107.

17. Reiber $H$, Lange $P$ : Quantification of virus-specific antibodies in cerebrospinal fluid and serum: sensitive and specific detection of antibody synthesis in brain. Clin Chem 1991, 37:1153-1160.

18. Reiber H, Peter JB: Cerebrospinal fluid analysis: disease-related data patterns and evaluation programs. J Neurol Sci 2001, 184:101-122.

19. Reiber H: Flow rate of cerebrospinal fluid (CSF)-a concept common to normal blood-CSF barrier function and to dysfunction in neurological diseases. J Neurol Sci 1994, 122:189-203.

20. Jarius S, Aboul-Enein F, Waters P, Kuenz B, Hauser A, Berger T, Lang W, Reindl M, Vincent A, Kristoferitsch W: Antibody to aquaporin-4 in the long-term course of neuromyelitis optica. Brain 2008, 131:3072-3080

21. Bergamaschi R: Importance of cerebrospinal fluid examination in differential diagnosis of Devic's neuromyelitis optica by multiple sclerosis. Neurol Sci 2003, 24:95-96.

22. Ghezzi A, Bergamaschi R, Martinelli V, Trojano M, Tola MR, Merelli E, Mancardi L, Gallo P, Filippi M, Zaffaroni M, Comi G: Clinical characteristics, course and prognosis of relapsing Devic's Neuromyelitis Optica. J Neurol 2004, 251:47-52

23. Zaffaroni M: Cerebrospinal fluid findings in Devic's neuromyelitis optica. Neurol Sci 2004, 25(Suppl 4):S368-370.

24. Melamud L, Madalena L, Facio ML, A PM, Garcea O, VA M: Cerebrospinal fluid findings in Devic's neuromyelitis optica. Multiple Sclerosis 2006, 12: P246.

25. O'Riordan Jl, Gallagher HL, Thompson AJ, Howard RS, Kingsley DP, Thompson EJ, McDonald WI, Miller DH: Clinical, CSF, and MRI findings in Devic's neuromyelitis optica. J Neurol Neurosurg Psychiatry 1996, 60:382-387.

26. Matiello M, Lennon VA, Jacob A, Pittock SJ, Lucchinetti CF, Wingerchuk DM, Weinshenker BG: NMO-IgG predicts the outcome of recurrent optic neuritis. Neurology 2008, 70:2197-2200.

27. Weinshenker BG, Wingerchuk DM, Vukusic S, Linbo L, Pittock SJ, Lucchinetti CF, Lennon VA: Neuromyelitis optica lgG predicts relapse after longitudinally extensive transverse myelitis. Ann Neurol 2006, 59:566-569.

28. Kinoshita M, Nakatsuji Y, Kimura T, Moriya M, Takata K, Okuno T, Kumanogoh A, Kajiyama K, Yoshikawa H, Sakoda S: Anti-aquaporin-4 antibody induces astrocytic cytotoxicity in the absence of CNS antigenspecific T cells. Biochem Biophys Res Commun 2010, 394:205-210.

29. Zhang ET, Inman CB, Weller RO: Interrelationships of the pia mater and the perivascular (Virchow-Robin) spaces in the human cerebrum. J Anat 1990, 170:111-123

30. Broadwell RD, Sofroniew MV: Serum proteins bypass the blood-brain fluid barriers for extracellular entry to the central nervous system. Exp Neurol 1993, 120:245-263.

31. Esiri MM, Gay D: Immunological and neuropathological significance of the Virchow-Robin space. J Neurol Sci 1990, 100:3-8.

32. Poduslo JF, Curran GL, Berg CT: Macromolecular permeability across the blood-nerve and blood-brain barriers. Proc Natl Acad Sci USA 1994, 91:5705-5709. 
33. Brimijoin $S$, Balm M, Hammond P, Lennon VA: Selective complexing of acetylcholinesterase in brain by intravenously administered monoclonal antibody. J Neurochem 1990, 54:236-241.

34. Saadoun $S$, Waters $P$, Bell BA, Vincent A, Verkman AS, Papadopoulos MC: Intra-cerebral injection of neuromyelitis optica immunoglobulin $\mathrm{G}$ and human complement produces neuromyelitis optica lesions in mice. Brain 2010, 133:349-361

35. Vincent T, Saikali P, Cayrol R, Roth AD, Bar-Or A, Prat A, Antel JP: Functional consequences of neuromyelitis optica-lgG astrocyte interactions on blood-brain barrier permeability and granulocyte recruitment. J Immunol 2008, 181:5730-5737.

36. Hinson SR, Pittock SJ, Lucchinetti CF, Roemer SF, Fryer JP, Kryzer TJ, Lennon VA: Pathogenic potential of IgG binding to water channel extracellular domain in neuromyelitis optica. Neurology 2007, 69:2221-2231.

37. Hinson SR, Roemer SF, Lucchinetti CF, Fryer JP, Kryzer TJ, Chamberlain JL, Howe CL, Pittock SJ, Lennon VA: Aquaporin-4-binding autoantibodies in patients with neuromyelitis optica impair glutamate transport by downregulating EAAT2. J Exp Med 2008, 205:2473-2481.

38. Zhou J, Kong H, Hua X, Xiao M, Ding J, Hu G: Altered blood-brain barrier integrity in adult aquaporin-4 knockout mice. Neuroreport 2008, 19:1-5.

39. Wingerchuk DM, Lennon VA, Pittock SJ, Lucchinetti CF, Weinshenker BG Revised diagnostic criteria for neuromyelitis optica. Neurology 2006, 66:1485-1489.

40. McDonald WI, Compston A, Edan G, Goodkin D, Hartung HP, Lublin FD, McFarland HF, Paty DW, Polman CH, Reingold SC, et al: Recommended diagnostic criteria for multiple sclerosis: guidelines from the International Panel on the diagnosis of multiple sclerosis. Ann Neurol 2001, 50:121-127.

doi:10.1186/1742-2094-7-52

Cite this article as: Jarius et al: Cerebrospinal fluid antibodies to aquaporin-4 in neuromyelitis optica and related disorders: frequency, origin, and diagnostic relevance. Journal of Neuroinflammation 2010 7:52.

\section{Submit your next manuscript to BioMed Central and take full advantage of:}

- Convenient online submission

- Thorough peer review

- No space constraints or color figure charges

- Immediate publication on acceptance

- Inclusion in PubMed, CAS, Scopus and Google Scholar

- Research which is freely available for redistribution

Submit your manuscript at www.biomedcentral.com/submit
Biomed Central 